\title{
Statistical Fracture Criterion of Brittle Materials Under Static and Repeated Loading
}

\author{
Dmytro Babich, ", Volodymyr Bastun ${ }^{2}$ \\ ${ }^{1}$ Department of Electroelasticity, Stepan Timoshenko Institute of Mechanics, National Academy of Sciences of Ukraine, Kyiv, Ukraine \\ ${ }^{2}$ Department of Fracture Mechanics of Materials, Stepan Timoshenko Institute of Mechanics, National Academy of Sciences of Ukraine, \\ Kyiv, Ukraine
}

Email address:

Babich_dv@ukr.net (D. Babich)

${ }^{*}$ Corresponding author

To cite this article:

Dmytro Babich, Volodymyr Bastun. Statistical Fracture Criterion of Brittle Materials Under Static and Repeated Loading. American Journal of Modern Physics. Vol. 6, No. 6, 2017, pp. 117-121. doi: 10.11648/j.ajmp.20170606.11

Received: August 2, 2017; Accepted: August 23, 2017; Published: September 19, 2017

\begin{abstract}
A statistical strength criterion for brittle materials under static and repeated loadings is proposed. The criterion relates beginning of a macrofracture in the form of origination of microcracks to the moment at which the microcrack density in the material becomes critical. The idea of the criterion consists in identification of the values of microdefect concentration under static and repeated loadings with the value of microdefect concentration which is held in the case of fracture under uniaxial static loading. It is assumed that the microcrack concentration defines the life of structures made of brittle materials. The numerical example of practical use of the criterion under consideration is presented.
\end{abstract}

Keywords: Statistical Strength Criterion, Brittle Materials, Static and Repeated Loadings, Microcrack Concentration

\section{Introduction}

A large body of studies reviewed in monographs [1]-[5], a.o. shows that fatigue failure of materials is a complex multiple-stage process which includes dispersed microfailure of structural elements. This is attributed to the fact that engineering materials contain randomly scattered over a volume microdefects, which under cyclic loading initiate microcracks. Later on these microdefects coalescence, that leads to formation of macrocracks and to the loss of the body integrity. The inherent random nature of fatigue failure requires a probabilistic analytical treatment to allow the prediction of a structural component's life and demonstrate the ability of a structure to maintain a specified strength for a certain period of use after sustaining damage [6], [7].

The prediction methods are based on various models that are outlined in [8]-[15] and involve the weakest link model, the linear damage accumulation rule, as well as such representations as the stress-life, Coffin-Manson, ParisErdogan ones. In the last case the fracture mechanics is used with a power law relationship between the crack growth rate and stress intensity factor. Approaches to the life assessment based on the use of continuum-damage mechanics and fracture-mechanics models are outlined in [16], [17]. The capability to predict the high cycle fatigue properties of adhesive joints is considered in [18].

In the present paper, a new probabilistic model of fatigue failure of structures made of brittle materials undergoing cyclic loading in the range of elastic strains is addressed. In accordance with the model, the beginning of macrofailure is related to the critical value of microcrack concentration and is defined by the statistical strength criterion whose nature is attributed to the probabilistic character of the microfailure process. It is assumed that the macrofailure occurs by forming macrocracks due to accumulation of microdefects in the form of flat microcracks randomly dispersed over a volume ([19]).

\section{Model of Microcrack Accumulation}

To describe the process of microfailure, the structural Daniels model of damage accumulation is involved. The physical meaning of the model as applied to a structurally inhomogeneous medium is clarified in [2]. As a criterion for failure of structural elements by rupture, we adopt the 
following:

$$
\bar{\sigma}_{33}^{\prime} \geq \sigma
$$

where $\bar{\sigma}_{33}^{\prime}$ is the local true stress, which refers to the undamaged part of the body cross-section, $\sigma$ is the stochastic variable associated with the limiting value of the true tensile or compressive normal stresses for arbitrarily oriented structural elements. To approximate the strength distribution of crystallites and grains with different orientations in microinhomogeneous materials, a power law is used. In this case the integral function of microstrength distribution $F(\sigma)$ is described as

$$
F_{i}(\sigma)=\left(\frac{\sigma}{\sigma_{i}}\right)^{\alpha_{i}}
$$

where

$$
\alpha_{i}=-1+\frac{1}{k_{w i}} \sqrt{1+k_{w i}^{2}}, \quad \sigma_{i}=\frac{\sqrt{1+k_{w i}^{2}}}{\sqrt{1+k_{w i}^{2}}-k_{w i}} \sigma_{u s i}^{\prime}
$$

Here $k_{w i}$ is the coefficient of variation:

$$
k_{w i}=\frac{\sqrt{D_{i}}}{\sigma_{u s i}^{\prime}}=\sqrt{\frac{1}{\alpha_{i}\left(2+\alpha_{i}\right)}} .
$$

$\sigma_{u s i}^{\prime}$ is the average value of the ultimate strengths of structural elements, $D_{i}$ is the dispersion of the ultimate microstrength, $\sigma_{i}$ and $\alpha_{i}$ are the parameters of the scatters of microstrength.

The integral function $\varepsilon_{i}=F_{i}(\sigma)$ of the microstrength distribution designates the fraction of the unit area of the random body section, in which the ultimate microstrength is less than a certain fixed value $\sigma$. This fraction is the total area of the sections of structural elements, where ultimate strengths are less than the value of the acting normal stress that causes their cracking.

The structural elements will failure when the stress $\bar{\sigma}_{33}^{\prime}$ reaches the limiting value of $\sigma$. Failure of individual elements gives rise to origin of population of random events. Interaction of the elements lies in the fact that after failure of the fraction of them stresses redistribute between nonfailed ones. When the true tensile normal stress $\bar{\sigma}_{33}^{\prime}$ reaches limiting value, these elements fail by forming microcracks with the planes being normal to the direction in which the stress $\bar{\sigma}_{33}^{\prime}$ acts. In the case of compressive stresses microcracks are oriented mainly in parallel with the direction of action of the stress $\bar{\sigma}_{33}^{\prime}$ [2],[19]. Since the failed structural elements resist to compression as solid ones, then $\bar{\sigma}_{33}^{\prime}=\sigma_{33}^{\prime}$. If the conditional local tensile stress $\sigma_{33}^{\prime}$ is considered as an independent loading parameter, then the true local stress in the sections of the nonfailed structural elements is defined approximately within the framework of the model under consideration by

$$
\bar{\sigma}^{\prime}=\frac{\sigma_{33}^{\prime}}{1-F_{1}\left(\bar{\sigma}_{33}^{\prime}\right)}
$$

Note that the true local stress $\bar{\sigma}_{33}^{\prime}$ and the given average stresses $\sigma_{k l}$ are related as follows ([19]):

$$
\sigma_{33}^{\prime}=\sigma_{k l} \alpha_{3 k} \alpha_{3 l}
$$

Here $\alpha_{3 k}$ and $\alpha_{3 l}$ are the direction cosines of the local coordinate system expressed through the angles $\vartheta$ and $\psi$ $(0 \leq \vartheta \leq \pi, \quad 0 \leq \psi \leq 2 \pi)$ by

$$
\alpha_{31}=\sin \psi \sin \vartheta, \alpha_{32}=-\cos \psi \sin \vartheta, \alpha_{33}=\cos \vartheta
$$

The volume concentration of flat microcracks is determined by the ratio of a number of the destroyed structural elements $N_{0 i}$ in tension or compression to their total number $N\left(p_{i}=N_{0 i} / N\right)$ in a representative volume. Using the method proposed by Kendall and Moran, 1963, which is common in petrography in analyzing thin sections of sediments, it can be shown that $p_{i}=\varepsilon_{i}$.

To determine the concentration of the cracked structural elements, the distribution parameters $\alpha_{i}$ and $\sigma_{i}$, which are expressed in terms of average values of the ultimate strengths and coefficient of variation $\mathrm{k}$, are used. Since dimensions of the structural elements are small, these parameters can not be determined directly. Because of this, they should be defined by using experimental magnitudes of conditional macrostrength parameters for the sample of macrospecimens $\left(\sigma_{u s i}, D_{i}^{2}, k_{w i}\right)$. Note that the coefficients of microstrength variation for structural elements expressed as relative values in terms of the conditional and true stresses are close in magnitudes. For this reason, the parameters $\alpha_{i}$ appearing in the functions of the macrostrength distribution for the material of specimens and structural elements coincide and are defined by the first formula from (3). In this case only the parameter $\sigma_{1}$ should be determined.

In compression ( $\left.\sigma_{i i}<0, i=1,2,3\right)$, these parameters, due to coincidence of the conditional and true stresses, are determined by (3). In tension ( $\sigma_{i i}>0, i=1,2,3$ ), only the parameter $\sigma_{1}$ should be determined by using the following procedure. According to (5), the average limiting value of the independent loading parameter (conditional stress) $\sigma_{u s 33}^{\prime}$ is determined in terms of the true stresses by

$$
\left\langle\sigma_{u s 33}^{\prime}\right\rangle=\bar{\sigma}_{33 \max }^{\prime}\left[1-F_{1}\left(\bar{\sigma}_{33 \max }^{\prime}\right)\right],
$$

where $\bar{\sigma}_{33 \max }^{\prime}$ is the maximum value of the stress $\sigma_{33}^{\prime}$ in the structural element, which is reached when specimens fail. Considering (2), (8), and the condition 


$$
\frac{d}{d \sigma}\left\{\sigma\left[1-F_{1}(\sigma)\right]\right\}=0 \text { at } \sigma=\bar{\sigma}_{33 \max }^{\prime}
$$

we get

$$
\bar{\sigma}_{33 \max }^{\prime}=\left(\frac{1}{1+\alpha_{1}}\right)^{\frac{1}{\alpha_{1}}} \sigma_{1}=\left\langle\sigma_{u s 33}^{\prime}\right\rangle \frac{1+\alpha_{1}}{\alpha_{1}} .
$$

Since $<\sigma_{u s 33}^{\prime}>=\sigma_{u s 1}$, then

$$
\sigma_{1}=\frac{\sigma_{u s 1}\left(1+\alpha_{1}\right)^{\frac{1}{\alpha_{1}+1}}}{\alpha_{1}}
$$

Note that the value $\left\langle\sigma_{u s 33}^{\prime}\right\rangle=\sigma_{u s 1}$ appearing in (10) and (11), is the average magnitude of the ultimate strength of the material, $\sigma_{1}$ are the maximum values of the strength of individual structural elements that is realized in failed specimens.

In what follows, we will consider a statistical strength criterion.

\section{Statistical Strength Criterion}

Let the stresses $\sigma_{i j}(i, j=1,2.3)$ be given in a laboratory coordinate system pertaining to the representative volume of the body. Then the microcrack concentration in the random section of a solid will be determined as follows:

$$
\varepsilon_{i}=F_{i}\left(\bar{\sigma}_{33}^{\prime}\right)=\left(\frac{\bar{\sigma}_{33}^{\prime}}{\sigma_{i}}\right)^{\alpha_{i}}(i=1,2),
$$

where the local stress $\bar{\sigma}_{33}^{\prime}$ in tension $\left(\sigma_{i j}>0\right)$ and compression $\left(\sigma_{i j}<0\right)$ is defined by

$$
\bar{\sigma}_{33}^{\prime} \approx \frac{\sigma_{33}^{\prime}}{1-F_{1}\left(\bar{\sigma}_{33}^{\prime}\right)}, \quad \bar{\sigma}_{33}^{\prime}=\sigma_{33}^{\prime}
$$

Here $\sigma_{33}^{\prime}$ is the conditional local stress (normal to the plane of a random section), which is determined in terms of the stresses given in the laboratory coordinate system by formulas (6) and (7).

Then the statistical strength criterion becomes:

$$
F_{i}\left(\bar{\sigma}_{33 m}^{\prime}\right) \leq \varepsilon_{i c r}(i=1,2),
$$

where $F_{i}\left(\bar{\sigma}_{33 m}^{\prime}\right)=\varepsilon_{i m}$ is the crack concentration in the section with the local normal true stress being maximum. If the conditional tensile stresses reach value $\sigma_{33 m}^{\prime}$, the concentration will be determined by

$$
\varepsilon_{1 m}\left(1-\varepsilon_{1 m}\right)^{\alpha_{1}}=\left(\frac{\sigma_{33 m}^{\prime}}{\sigma_{1}}\right)^{\alpha_{1}}
$$

In compression, due to coincidence of true and conditional stresses, $\varepsilon_{2 m}=\left(\frac{\sigma_{33 m}^{\prime}}{\sigma_{2}}\right)^{\alpha_{2}}$.

The critical values of the microcrack concentration $\varepsilon_{i c r}$, which appear in (14), under tension or compression are determined as follows:

$$
\varepsilon_{1 c r}\left(1-\varepsilon_{1 c r}\right)^{\alpha_{1}}=\left(\frac{\sigma_{u s 1}}{\sigma_{1}}\right)^{\alpha_{1}}, \quad \varepsilon_{2 c r}=\left(\frac{\sigma_{u s 2}}{\sigma_{2}}\right)^{\alpha_{2}}
$$

Here $\sigma_{i c r}(i=1,2)$ are the average values of the ultimate strengths in tension and compression, respectively.

It should be noted that the rate of accumulation of microcracks in a material depends on how loading is realized (frequency, rate, etc.). Assume that before deforming the material had initial microdamages of density $\varepsilon_{i 0}$. The distribution function $F_{i}(\sigma)$ appearing in (2) defines the relative fraction of remaining nonfailed structural elements with area $\left(1-\varepsilon_{i 0}\right)$ whose ultimate strength is either equal or less than some value of $\sigma$. For this reason, since in the nonfailed fraction of the section $\bar{\sigma}_{33}^{\prime}>\sigma$, the function $F_{i}\left(\bar{\sigma}_{33}^{\prime}\right)$ will evaluate the relative content of destroyed microelements in the remaining part of the nonfailed section $\left(1-\varepsilon_{i 0}\right)$. In this case, the microcrack concentration in the random section of the solid will be determined, with the stress increasing under monotonic (static) loading to $\bar{\sigma}_{33}^{\prime}$, by

$$
\varepsilon_{i}=\varepsilon_{i 0}+\left(1-\varepsilon_{i}\right) F_{i}\left(\bar{\sigma}_{33}^{\prime}\right)=\varepsilon_{i 0}+\left(1-\varepsilon_{i}\right)\left(\frac{\bar{\sigma}_{33}^{\prime}}{\sigma_{i}}\right)^{\alpha_{i}}(i=1,2)
$$

Then relations (15) become

$$
\left(\varepsilon_{1 c r}-\varepsilon_{10}\right)\left(1-\varepsilon_{1 c r}\right)^{\alpha_{1}-1}=\left(\frac{\sigma_{u s 1}}{\sigma_{1}}\right)^{\alpha_{1}}, \quad \varepsilon_{2 c r}=\varepsilon_{20}+\left(1-\varepsilon_{2 c r}\right)\left(\frac{\sigma_{u s 2}}{\sigma_{2}}\right)^{\alpha_{2}}
$$

Note that similar approach to determination of microcrack concentration was employed in [20]. Below we will consider 


\section{Modeling the Fatigue Failure}

The possibility for the above criterion to be used in the case of cyclic loading is based on the experimentally established relationship of the mechanisms of fatigue failure and microcrack accumulation in a material under repeated loading. However, it is necessary to keep in mind that in this case the mechanisms of microfailure in tension and compression are dissimilar.

Assume that a specimen made of a defect-free material $\left(\varepsilon_{1(0)}=0\right)$ undergoes uniaxial repeated tension with a stress $\sigma_{330}$. Then, in accordance with (16) after the first cycle $((n)=(1))$ of loading, damages origin with concentration

$$
\varepsilon_{1(1)}=\left(1-\varepsilon_{1(1)}\right)^{1-\alpha_{1}}\left(\frac{\sigma_{330}}{\sigma_{1}}\right)^{\alpha_{1}}
$$

As a result of $n$ - fold tension, the density of damaged elements in the specimen cross-section increases and is determined by

$$
\varepsilon_{1(n)}=\varepsilon_{1(n-1)}+\left(1-\varepsilon_{1(n)}\right)^{1-\alpha_{1}}\left(\frac{\sigma_{330}}{\sigma_{1}}\right)^{\alpha_{1}}
$$

where $\varepsilon_{1(n-1)}$ is the concentration of microcracks, which have appeared after the $(n-1)$-fold tension. The fatigue failure of the specimen will occur at the $N$-th cycle of loading when the microcrack concentration reaches critical value $\varepsilon_{1(N)}=\varepsilon_{1 c r}$ defined by

$$
\begin{gathered}
\varepsilon_{1 c r}=\varepsilon_{1(N-1)}+\left(1-\varepsilon_{1 c r}\right)^{1-\alpha_{1}}\left(\frac{\sigma_{330}}{\sigma_{1}}\right)^{\alpha_{1}} \cdot(20) \\
\sigma_{u s 33}=\sigma_{1}\left(\varepsilon_{1 c r}-\varepsilon_{1(n)}\right)^{1 / \alpha_{1}}\left(1-\varepsilon_{1 k}\right)^{1-1 / \alpha_{1}} ; \sigma_{1 e}=\sigma_{1} \varepsilon_{1 c r}^{1 / \alpha_{1}}\left(1-\varepsilon_{1 c r}\right)^{1-1 / \alpha_{1}} / N_{0}^{1 /} \alpha_{1},
\end{gathered}
$$

The service life, which is characterized by a number of cycles $N$ to failure, can be determined either by using equation (19) or relation (20). The last variant is preferable since it does not require solving equation (19) of arbitrary power. At the same time, the necessity in successive calculations of intermediate values of the microcrack concentration arises. This is attributed to the fact that damaging action of successive cycles becomes stronger as microcracks are accumulated with increase in a number of loading cycles.

The approximate approach to defining the service life $N$ is related to determination, considering (19), of several $(k)$ values of increments of the microcrack concentration $\Delta_{i} \varepsilon_{i}$ for separate cycles of tension with successive determination of their average value. In this case the value of $N$ is defined by

$$
N=\varepsilon_{1 c r}\left[\left(\frac{\sigma_{330}}{\sigma_{1}}\right)^{\alpha} \times \frac{1}{k} \sum_{i=1}^{k}\left(1-\varepsilon_{1(i))}\right)^{1-\alpha_{1}}\right]^{-1},
$$

where $\varepsilon_{1(i)}=i \frac{\varepsilon_{1 c r}}{k}$ are the values of the microcrack concentration arranged in increasing order on the interval $\left[\varepsilon_{1(1)}, \varepsilon_{1 c r}\right]$.

The approach being considered makes it possible also to establish the residual ultimate strength of the material $\sigma_{u s 33}$, which has underwent $n$ cycles of loading, as well as the conditional endurance limit $\sigma_{1}$ at the given number of cycles $N_{0}$.

The associated expressions for the searched values are: where $\varepsilon_{1(n)}$ is the concentration of microcracks accumulated after the $n$-fold loading.

\section{Numerical Example}

Let us determine, as an example, the life of a rod which undergoes action of the periodically varying with time $t$ stress $\sigma_{-1}$. The rod is made of the 45 carbon steel (the content of $\mathrm{C}=0.45 \%$ ) whose average value of the ultimate strength $\sigma_{u s 1}$ and coefficient of variation $k_{w 1}$ are ([3]):

$$
\sigma_{u s 1}=674 M P a, \quad k_{w 1}=0.07 .
$$

The one end of the rod is clamped while other is free. Considering (3) and (11), we get:

$$
\sigma_{1}=885 M P a, \alpha_{1}=13,32 .
$$

Then, with (17), the critical value of the microcrack concentration $\varepsilon_{1 c r}=0.05$ is determined. Using (21), for $k=5$ and $\sigma_{-1}=280 \mathrm{MPa}$, it follows $N=15 \cdot 10^{4}$.

The corresponding experimental value of $N$ under certain conditions is equal to $17 \cdot 10^{4} \quad$ [3]. As can be seen, the theoretical value is in satisfactory agreement with experimental one.

\section{Conclusions}

A statistical strength criterion for brittle materials describing occurrence of critical state (fracture) under static and repeated loadings has been proposed. The criterion is 
formulated in the terms of a damage measure of brittle materials. As such one, the concentration of microdefects in the form of flat mode I microcracks stochastically dispersed over a volume was chosen. The criterion stated in combination with a structural model of accumulation of microdefects makes it possible to develop a technique for theoretical prediction of service life of structures made of these materials under repeated loading with allowance for peculiarities of their fracture.

\section{References}

[1] H. O. Fuchs and R. J. Stephens, Metal Fatigue in Engineering. John Wiley \& Sons, 1980.

[2] V. V. Bolotin, Machine and Structure Life Prediction. Mashinostroenie, Moscow, 1984, (in Russian).

[3] V. T. Troshchenko and L. A. Sosnovskii, Fatigue Strength of Metals and Alloys. Handbook in two parts. Part 1. Naukova Dumka, Kyiv, 1987 (in Russian).

[4] W. Schutz and P. Heuler, A review of fatigue life prediction models for the crack initiation and propagation phases, in: Advances in Fatigue Science and Technology (ed. by C. Moura Branco and L. Cuerra Rosa), 1989, pp. 177-219.

[5] L. Pook, Metal Fatigue, 2009, Springer.

[6] J. Luo and P. Bowen, A probabilistic methodology for fatigue life prediction, Acta Materiala, vol. 51 (12). 2003, pp. 3537 3550 .

[7] T. D. Righiniotis and M. K. Chryssanthopoulos, Probabilistic fatigue analysis under constant amplitude loading, J. of Constructional Steel Research, vol. 59 (7), 2003, pp. 867-886.

[8] K. Ortiz and A. S. Kiremidjian, Stochastic modeling of fatigue crack growth, Engineering Fracture Mechanics, vol. 29 (3), 1988, pp. 317-334.

[9] W. Schutz and P. Heuler, A review of fatigue life prediction models for the crack initiation and propagation phases, in: Advances in Fatigue Science and Technology (ed. by C. Moura Branco and L. Cuerra Rosa), 1989, pp. 177-219.
[10] G. Maymon, The problematic nature of the application of stochastic crack growth models in engineering design, Engineering Fracture Mechanics, vol. 53, (6), 1996, pp. 911916.

[11] W. Cui, A state-of-the-art review on fatigue life prediction methods for metal structures, J. of Marine Science and Technology, vol. 7 (1), 2002, pp. 43-56.

[12] W. F. Wu and C. C. Ni, Probabilistic models of fatigue crack propagation, Probabilistic Engineering Mechanics, 18 (3), 2004, pp. 247-257.

[13] D. V. Babich, O. I. Bezverkhyi, T. I. Dorodnykh, Continuum model of deformation of piezoelectric materials with cracks, Applied Mechanics and Materials, vol. 784, 2015, pp. 161-172.

[14] D. V. Babich, V. N. Bastun, and T. I. Dorodnykh, Structuralprobabilistic approach to determining the durability for structures of brittle materials, Acta Mechanics, vol. 228, 207, pp. 269-274.

[15] D. V. Babich D. V., T. I. Dorodnykh. Determining cyclic durability of piezoceramic structures using probabilistic approach, Key Engineering Materials, vol. 713, 2016, pp. 216219.

[16] Y. C. Xiao, S. Li. Z., K. Gao, A continuum damage mechanics model for high cycle fatigue, International Journal of Fatigue, vol. 20, (7), 1998, pp. 503-508.

[17] Y. S. Upadhyaya, B. K. Sridhara, Fatigue life prediction. A continuum damage mechanics and fracture mechanics approach, Materials \& Design 35, 2012, pp. 220-224.

[18] A. Eklind, T. Walander, T. Carlberger, U. Stigh, High cycle fatigue crack growth in mode I of adhesive layers: modeling, simulation and experiments, Int. J. of Fracture, vol. 190 (1-2), 2014, pp. 125-146.

[19] D. V. Babich and V. N. Bastun, On dispersed microdamageability of elastic-brittle materials under deformation, J. of Strain Analysis, vol. 45 (1), 2010, pp. 5766 .

[20] V. P. Tamuzh, and V. S. Kuksenko, Micromechanics of fracture of polymer materials, Zinatne, Riga, 1978 (in Russian). 